\section{Interlaboratory comparison of humic substances compositional space as measured by Fourier transform ion cyclotron resonance mass spectrometry (IUPAC technical report)}

\author{
Zherebker, A., Kim, S. et al. \\ Pure and Applied Chemistry, 2020 \\ published online ahead of print 18 Aug 2020 \\ https://doi.org/10.1515/pac-2019-0809
}

Interlaboratory comparison on the determination of the molecular composition of humic substances (HS) was undertaken in the framework of IUPAC project 2016015-2-600. The analysis was conducted using high resolution mass spectrometry, nominally, Fourier transform ion cyclotron resonance mass spectrometry (FTICR MS) with electrospray ionization. Six samples of HS from freshwater, soil, and leonardite were used for this study, including one sample of humic acids (HA) from coal (leonardite), two samples of soil HA (the sodpodzolic soil and chernozem), two samples of soil fulvic acids (FA) (the sod-podzolic soil and chernozem), and one sample of freshwater humic acids (the Suwannee River). The samples were analyzed on five different FTICR MS instruments using the routine conditions applied in each participating laboratory. The results were collected as mass lists, which were further assigned formulae for the determination of molecular composition.

The similarity of the obtained data was evaluated using appropriate statistical metrics. The results have shown that direct comparison of discrete stoichiometries assigned to the mass lists obtained by the different laboratories yielded poor results with low values of the Jaccard similarity score-not exceeding 0.56 (not more than $56 \%$ of the similar peaks). The least similarity was observed for the aromatics-rich HA samples from leonardite (coal) and the chernozem soil, which might be connected to difficulties in their ionization. The reliable similarity among the data obtained in this intercomparison study was achieved only by transforming a singular point (stoichiometry) in van Krevelen diagram into a sizeable pixel (a number of closely located stoichiometries), which can be calculated from the population density distribution. The conclusion was made that, so far, these are descriptors of occupation density distribution, which provide the metrics compliant with the data quality requirements, such as the reproducibility of the data measurements on different instruments.

https://iupac.org/project/2016-015-2-600

\section{Structure, processing and performance of ultra-high molecular weight polyethylene (UHMWPE) (IUPAC Technical Report) (4 parts)}

Part 1: characterizing molecular weight

Part 2: crystallinity and supra molecular structure

Part 3: deformation, wear and fracture

Part 4: sporadic fatigue crack propagation

Clive Bucknall, Volker Altstädt, et al.

Pure and Applied Chemistry, 2020

published online ahead of print 24 Aug 2020

https://doi.org/10.1515/pac-2019-0405 (part 1);

https://doi.org/10.1515/pac-2019-0403 (part 2);

https://doi.org/10.1515/pac-2019-0406 (part 3); and

https://doi.org/10.1515/pac-2019-0408 (part 4)

The aim of this project was to study the efficacy of current methods of quality control and quality assurance for ultra-high molecular weight polyethylene (UHMWPE) products, and find improvements where possible. Intrinsic viscosity tests were performed on three grades of polyethylene with weight average relative molar masses of about $6 \times 10^{5}, 5 \times 10^{6}$, and $9 \times 10^{6}$. Results from three laboratories showed substantial scatter, probably because different methods were used to make and test solutions. Tensile tests were carried out to $600 \%$ extension at $150{ }^{\circ} \mathrm{C}$ under both constant applied load and constant Hencky strain rate, on compression mouldings made by a leading manufacturer of ultra-high molecular weight polyethylene. They gave low values of $\bar{M}_{\mathrm{w}}$, suggesting incomplete entanglement at 'grain boundaries' between powder particles. Results from conventional melt-rheology tests are presented, and their relevance to quality control and assurance is discussed. Attempts to calculate molecular weights from these data met with limited success because of extended relaxation times. Suggestions are made for improving international standards for intrinsic viscosity testing of UHMWPE, by investigating the various factors that can cause significant errors, and by introducing methods for checking the homogeneity (and hence validity) of the solutions tested.

Part 2 addresses characterization of crystallinity and structure. Part 3 covers mechanical properties, and Part 4 focuses on the sporadic crack propagation behavior exhibited by all three grades of UHMWPE in fatigue tests on $10 \mathrm{~mm}$ thick compact tension specimens. 